\title{
100GHz Electrically Tunable Planar Bragg Gratings via Liquid Crystal Overlay
}

\author{
F.R. Mahamd Adikan, J.C. Gates, B.D. Snow, A. Dyadyusha, H.E. Major, C.B.E. Gawith, M. Kaczmarek, P.G.R. Smith \\ University of Southampton, SO17 1BJ Southampton, United Kingdom
}

We demonstrate $114 \mathrm{GHz}$ electrically tunable liquid crystal Bragg gratings using $170 \mathrm{Vpp}$ voltage. The devices were made using direct UV grating writing and use evanescent coupling into an electrically tuned nematic liquid crystal.

Reconfigurable integrated optical devices are essential in today's dense and complex telecommunication meshes. A commonly employed component on the silica platform fulfilling the above role is a planar Bragg grating. The ability to tune the reflection peak of these gratings is one of the key enablers in realizing an all optical dynamic network. To date, little has been reported on electrically tunable planar Bragg gratings given their potentially superior response times over temperature tuned devices. Such electrically tunable devices work on the principle of shifting the Bragg wavelength by modifying the effective index of a waveguide in a multilayer substrate. One route to achieve this is by overlaying the grating with a liquid crystal as many liquid crystals display refractive index anisotropy that can be electrically manipulated. Modifying the liquid crystal refractive index subsequently alters the effective index of the waveguide, leading to Bragg wavelength shift. Using this approach, Sparrow et al [1] have previously demonstrated $35 \mathrm{GHz}$ tunability at $1560 \mathrm{~nm}$ using $80 \mathrm{Vpp}$ (peak-to-peak) square-wave with $250 \mu \mathrm{m}$-spaced aluminum electrodes. Here, we report a maximum tunability of $114 \mathrm{GHz}$ at $1561.8 \mathrm{~nm}$ using patterned ITO glass electrodes with $170 \mathrm{Vpp}$ voltage at 1 $\mathrm{kHz}$. Two distinct threshold behaviors which manifest only during the increase of supply voltage were also observed.

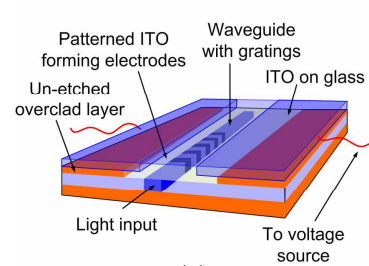

(a)

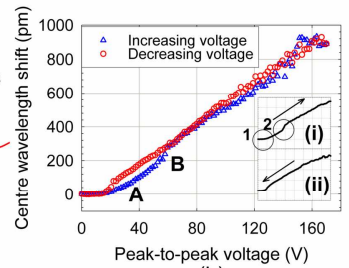

(b)

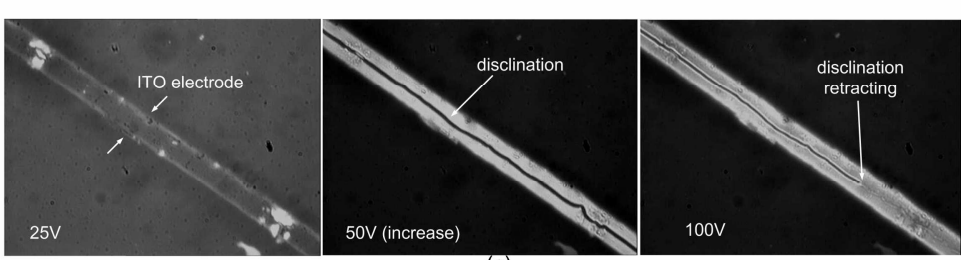

(c)

Figure 1(a) Schematic diagram of liquid crystal Bragg grating configuration; (b) Tuning curve with insets showing the threshold points; (c) Disclination lines seen via cross polarized microscope.

The samples used were 3-layer silica-on-silicon with a germanium doped core layer to allow photosensitivity to UV radiation. Waveguides and Bragg gratings were defined using Direct Grating Writing [2] and the overclad layer covering the gratings was subsequently removed using hydrofluoric acid wet etching. The electrodes were prepared by electrically scribing a $\sim 60 \mu \mathrm{m}$ gap into a piece of single sided Indium Tin Oxide (ITO) coated glass. Homeotropic alignment of the liquid crystal molecules was achieved by applying a thin layer of surfactant to the etched area and ITO electrodes. The electrodes were then attached to the top of the sample and Merck 18523 liquid crystal was inserted into the etched window under vacuum (Fig 1(a)). This liquid crystal is characterized by extraordinary and ordinary refractive index values of $1.49\left(\mathrm{n}_{\mathrm{e}}\right)$ and $1.44\left(\mathrm{n}_{\mathrm{o}}\right)$ at $1550 \mathrm{~nm}$ respectively. The grating spectrum and the centre wavelength shift were recorded for each voltage condition and Figure 1(b) shows the tuning curve of the device, exhibiting 932.7pm (114GHz at $\lambda=1561.8 \mathrm{~nm}$ ) Bragg wavelength shift with 170Vpp. Interestingly, the curve displays hysteresis between points A and B (Fig. 1(b)) where inset (i) shows the tuning curve with increase in voltage and inset (ii) shows the curve with decreasing voltage. It was observed that the curves for increasing voltage exhibit two distinct points where the tuning gradient increases significantly (circled). We believe that the lower threshold is where the electric field is large enough to overcome interactions of liquid crystal molecules with surfaces and elastic forces. The origin of the higher threshold is not clear, but is likely due to the multi-domain structure of liquid crystals created in the etched window and their interactions with applied field, creating disclination lines along a particular axis as shown in Fig. 1(c).

We will discuss design considerations for a wider span device and the use of disclination effects as potential routes towards high-speed switching devices. The demonstrated Bragg wavelength tuning over multiple telecoms channel spacing electrically is encouraging, and our development of a cascaded architecture of such integrated liquid crystal devices operating at different Bragg wavelengths could pave the way towards true colorless add/drop modules for dynamic dense optical networks.

[1] I. J. G. Sparrow, G. D. Emmerson, P. G. R. Smith, M. Kaczmarek, and A. Dyadyusha, "35 GHz tunable planar Bragg grating using nematic liquid crystal overlay," ECIO, Munich, Germany, 2005.

[2] G. D. Emmerson, C. B. E. Gawith, I. J. G. Sparrow, R. B. Williams, and P. G. R. Smith, "Physical observation of single step UV-written integrated planar Bragg structures and their application as a refractive-index sensor," Applied Optics, vol. 44, pp. 5042, 2005. 